\title{
Novel Environment Friendly Nanotechnologies
}

\author{
Oleg L Figovsky* and Blank N
}

Israel Association of Inventors, Israel

*Corresponding author: Oleg L Figovsky, Israel

Submission: 漈 October 26, 2018; Published: 些 October 29, 2018

\section{Editorial}

Our last elaboration-network nanostructured polymer system has been named a winner in the third annual NASA Nanotech Briefs $\AA^{\prime}$, Nano $50^{\mathrm{TM}}$ Awards in the Technology category.

Development projects currently underway in our center include the following:

1. Nanostructured composites based on Interpenetrated Polymer Network;

2. Epoxy-rubber \& epoxy - HUM composites with nanoheterogenic structure;

3. Nanocomposites based on hybrid organo-silicate matrix;

4. Polymer nanocomposites with very low permeability and high resistance to aggressive environments;

5. Metal matrix nanocomposites produced by SDP method.

6. Polymer matrix nanocomposites and nanomembranes produced by SDP metod;

Production of composite materials based on interpenetrating polymer networks provides a unique possibility to regulate their micro-and nano-structured properties. The new class of nanocomposite materials is characterized by the absence of contaminants at polymers production. The main components of the technology are branched (dendro)-aminosilanes which serve as curing agents for many oligomers. Additional hydrolysis of aminosilane oligomers creates the secondary nano-structured network polymer that improves service properties of the compound.

We have elaborated a number of nanostructured composites based on different kinds of soluble silicates during the liquid phase technological process. Significant increasing of silicate matrix strength and toughness was reached by incorporation of special liquid additives, such as TFS are acting as a microcrystallizing nucleator. Among our last development it is necessary to note a novel type of soluble silicate contained organic cations, for example, the DABCO based organic alkali soluble silicate.

Production of novel metallic matrix nanocomposites is based on the new physical effect "superdeep penetration" (SDP). These materials can be used for metal-cutting and stamp tools, for cutters of coal and mining machines instead of usual tool steel or special

alloys. Service life of the novel steel matrix nanocomposites tools is 1.5-5.0 times greater than usual tool. SDP technology can be applied for volumetric strengthening any type of cutting tool steels. SDP effect allows to produce anisotropic nanocomposite materials with the aluminum matrix. Electro conductivity of these materials in mutual perpendicular directions can differ twice. The new SDP technology of volumetric reorganization of aluminum matrix will find wide application in manufacture of electric installations and electronic devices.

A new way of modification of epoxy-amine compositions is discussed. It was synthesized hydroxyurethane modifier (HUM) on the base of primary polyamines and cyclocarbonates. Synthesized HUM was used for modification of different epoxy-amine compositions. The modifier does not react with epoxy components and it is not a part of the polymer matrix. The HUM is compatible with all types of epoxy resins. The HUM (5-40\% of epoxy component) may be introduced into a base of curable epoxy -amine composition by mixing. The HUM does not plasticate epoxy-amine composition does not change water resistant and accelerate curing. It was found that modified compositions have high chemical resistance and increased mechanical properties and wear abrasion. The HUM may be used as levelling component. High chemical resistance and increased mechanical properties probably depends on intermolecular hydrogen bonds of the HUM and the hydrogen bonds of the matrix.

Conventional disposable packaging and service items such as containers, trays, plates, bowls, and others, are commonly made from polystyrene or other synthetic hydrophobic plastics and also from paper or paperboard coated commonly with polyethylene. These materials are durable and are moisture-resistant and grease-resistant. The items are produced by industry in great quantities and are relatively inexpensive. After usage, these biostable packaging items are discarded in the environment and therefore create serious ecological problems. The expected lifetime of polystyrene packaging, for example, is approximately 500 years. Further, polystyrene and other plastics are made from nonrenewable petroleum resources. Recycling of plastic items is an expensive process. Moreover, recycled plastics are prohibited as a material for production packaging for food.

The pressure to use biodegradable disposable packaging materials has steadily increased in the last decade. The main approach in 
the art has been the manufacture of compostable and inexpensive food-service items such as containers, trays, plates and bowls based on biodegradable and natural raw materials such as starch, cellulose, proteins, etc. However, packaging based on natural biodegradable materials has several disadvantages, the most important being susceptibility to water, grease, and various other liquids. A starch binder is water-soluble and penetrable to grease. Cellulose and proteins are hydrophilic and therefore swell in water. When exposed to water or significant amounts of water vapor, these packaging items lose form-stability and become susceptible to breakage. Water, grease, and other liquids, as well as gases and vapors, easily penetrate natural materials because of high porosity of these materials.

To solve the susceptibility of natural materials to penetration of water and other liquids we proposed an idea to fill the pores on the surface of the natural biodegradable packaging material with fine barrier particles. Because paper and other natural packaging materials contain micron-scale pores, filling of these micro-pores with biodegradable nano-scale particles provides closing the pores and thus makes the natural materials stable against penetration of water, greases and other liquids [1-3].

Biodegradable nano-particles of cellulose in combination with some other additives appeared to be most suitable for the purposes of this elaboration. New method for obtaining of nano-cellulose particles was developed. This method comprises selective oxidative pretreatment of cellulose raw-material, controlled hydrolysis and high-pressure disintegration into nano-scale particles (20x200nm).

We have elaborated a number of nanostructured composite coatings based on different kinds of soluble silicates during the liquid phase technological process. Significant increasing of silicate matrix strength and toughness was reached by incorporation of special liquid additives, such as TFS which are acting as a microcrystallizing nucleator. Among our last development it is necessary to note a novel type of soluble silicates contained organic cations, for example, the DABCO based organic alkali soluble silicate.

Novel chemically resistant polymer coatings were elaborated through incorporation the nano-size inorganic active fillers. These nanoparticles react with aggressive medium, forming a new phase of high-strength hydrate complexes. The chemical resistant properties of the forming polymer materials are activated by harsh environmental conditions where polymer systems without additives remain defenseless to chemical corrosion.

New technology for production of polymeric nanomembranes is elaborating by SDP-method. Nanopores are creating by forming high soluble nonorganic salts, such as $\mathrm{Na}_{2} \mathrm{O}$, with following washing away by a distilled water.

\section{References}

1. Figovsky O, Beilin D (2013) Advanced polymer concretes and compounds. CRC Press, USA, pp. 1-272.

2. Kudryavtsev P, Figovsky O (2004) Nanomaterials based on soluble silicates. Lambert AC, USA, pp. 1-248.

3. Figovsky O, Beilin D (2017) Green Nanotechnology. Pan Stanford Publishing, USA, pp. 1-538.
Creative Commons Attribution 4.0 International License

For possible submissions Click Here

\section{Submit Article}

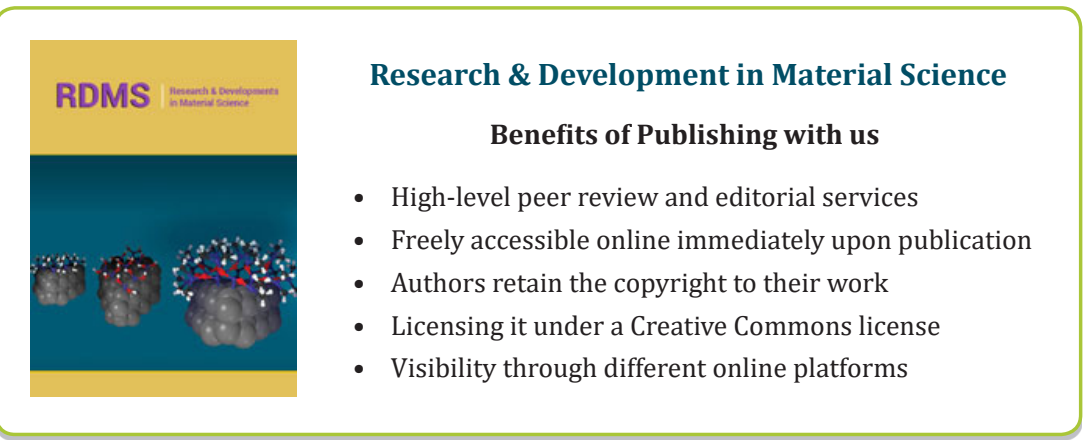

